\title{
Efecto hipolipemiante del aceite de Sacha Inchi en adultos con hipercolesterolemia e hipertrigliceridemia de 35 - 64 años, Nuevo AA.HH. Nueva Alianza, Chaclacayo, Lima
}

\author{
Lipid-lowering effect of Sacha Inchi oil in adults with hypercholesterolemia and hypertriglyceridemia \\ in 35-64 years old. AA.HH. Nueva Alianza, Chaclacayo. Lima
}

María Gamarra Camachoํㅜ, Betzabé Flores Albino², Félix Palacios Morales ${ }^{3}$

\begin{abstract}
RESUMEN
Objetivo: Determinar el efecto hipolipemiante del aceite de Sacha Inchi (Plukenetia volúbilis linneo) administrado en personas con hipercolesterolemia e hipertrigliceridemía de 35 a 64 años en el AA. HH. Nueva Alianza Chaclacayo. Material y métodos: Estudio de diseño preexperimental, de corte longitudinal prospectivo y de alcance explicativo. El grupo de participantes se conformó de 30 personas, en quienes se determinaron los valores sanguíneos de colesterol total (CT), colesterol (LDL), colesterol (HDL) y triglicéridos (TG). Los participantes recibieron $45 \mathrm{ml}$ de aceite de Sacha Inchi por día durante 42 días. El Requerimiento Diario Admitido (RDA) de Omega-3 para una persona entre las edades 35 a 64 años es el 1.5\% del valor calórico total del día. Teniendo como base una dieta de 1700 calorías, según el recordatorio de alimentos realizados a los participantes. Resultados: $\mathrm{Al}$ término del tratamiento, el grupo experimental tuvo diferencia significativa comparados con los resultados presentados al inicio de la intervención $(\mathrm{p}<0.05)$. Conclusiones: La ingestión diaria, de aceite Sacha Inchi, disminuye la hipercolesterolemia e hipertrigliceridemia.
\end{abstract}

Palabras clave: Aceite Sacha Inchi, Omega 3 (W3), colesterol total (CT), colesterol (LDL), colesterol (HDL), triglicéridos (TG).

\begin{abstract}
Objective: Determine the lipid lowering effect of the Sacha Inchi oil (Plukenetia volúbilis Linneo) administrating to people who have hypercholesterolemia and hypertriglyceridemia of 35 to 64 year old in the AA.HH. Nueva Alianza-Chaclacayo. Methods: The study is pre-experimental design of prospective longitudinal cut and explanatory model. The group of patients was conformed by 30 people, who were determined blood samples of total cholesterol (CT), cholesterol (LDL), cholesterol (HDL) and triglycerides (TG). The treatment was consisted in adminitrate $45 \mathrm{ml}$ of Sacha Inchi oil per day, during a month and a half, knowing that the admitted daily request (ADR) of omega 3 (w3) for a person who is 35 to 64 years old, is $1.5 \%$ of the total heat value of the day in a diet rich in 1700 calories, according to the reminder of food realized by the participants. Results: At the end of the treatment, the experimental group had favorable significant differences compare with the presented results at the beginning of the intervention. These results are the decrease of the levels of total cholesterol, cholesterol LDL, triglycerides and increase of cholesterol HDL. Conclusions: In conclusion the daily ingestion of sacha inchi oil, decrease the hypercholesterolemia and hypertriglyceridemia in people of 35 to 64 years old.
\end{abstract}

Key words: Oil of Sacha Inchi seed, total cholesterol (TC), cholesterol (LDL), cholesterol (HDL), triglycerides (TG)

${ }^{1}$ Jefa de Laboratorio de Nutrición, Universidad Peruana Unión. Lima, Perú.

${ }^{2}$ Nutricionista del Colegio Mayor Secundario Presidente del Perú. Lima, Perú.

${ }^{3}$ Jefe de Laboratorio de Biología. Universidad Peruana Unión. Lima, Perú. 


\section{INTRODUCCIÓN}

Actualmente la humanidad se ha distanciado de la alimentación saludable, trayendo como consecuencia enfermedades crónicas como ateroesclerosis, hipertensión arterial, obesidad, hiperlipidemias, diabetes, artritis y otras enfermedades autoinmunes y muchos cánceres, especialmente de mama, colon y próstata. En el Perú se calcula que 1 millón de personas sufren cada año su primer infarto de miocardio, de ellos sobreviven 400.000, de los cuales fallecen 50.000 al año siguiente por otro episodio agudo; la patología subyacente a estas afecciones, la ateroesclerosis, se constituye entonces en un área de interés común a la mayor parte de las dislipidemias médicas (NCEP, 2001).

Las enfermedades cardiovasculares representan la primera causa de muerte en el mundo. Se ha demostrado que el riesgo coronario aumenta con la edad y el tipo de régimen dietético, alto en grasas saturadas, según las previsiones de la Organización Mundial de la Salud. Por supuesto hay que considerar, en adición a la dieta, el estilo de vida sedentario y exposición a sustancias nocivas que interaccionan con procesos bioquímicos controlados genéticamente a enfermedades crónicas (Simopoulos, 2008).

Estudios recientes han mostrado que alimentos ricos en omega tiene efecto hipolipemiante, como el estudio que se hizo en CAMEC-IQUITOS, seguro social de salud, buscó establecer el efecto del aceite de Sacha Inchi Plukenetia volubilis $L$. Sobre la hipercolesterolemia e hipertrigliceridemia, concluyendo que la ingestión diaria, de aceite Sacha Inchi, disminuye la hipercolesterolemia e hipertrigliceridemia (Cordova M, 2008)). Otro estudio experimental, hecho en la ciudad de Trujillo, buscaba determinar el efecto de las semillas de Sacha Inchi en el perfil lipídico. Concluyendo que el consumo de Sacha Inchi Plukenetia volubilis L. reduce los niveles de triglicéridos, colesterol total, LDL y aumenta los niveles de HDL en adultos jóvenes (Gorriti A, 2010).

El estudio, realizado por Ramón y cols (Ramón Segura, 2007), usó como fuente de omega 3 el pescado azul en personas normales como en pacientes con niveles de colesterol HDL bajos, concluyendo que la ingesta mostró, a las 6 semanas, un aumento de $30.2 \%$ y en el 3er mes $47.71 \%$, (Harris WS, 1983) demostró que después de 4 semanas con una dieta rica de salmón encontraron un aumento del $50 \%$ en el colesterol HDL.

El sacha inchi (Plukenetia volubilis Linneo) viene de la familia Euphorbiaceae, y en el Perú es reconocida por presentar 61 géneros y 323 especies (Brako \& Zarucchi; Ulloa Ulloa et al., 2004), Asimismo, se reconocen otras tres especies, $P$. polyandenia Muell., P.loretensis Ule y P. brachybotrya Muell; el 2009 fue reportada una nueva especie Plukenetia huallabambana procedente de Amazonas (Bussman $\mathrm{R}, 2009$ ).

El Sacha Inchi es originario de la provincia de San Martín - Perú (Correo \& Yesid, 2007), la cual posee semillas de la que se obtiene aceite, harina de uso frecuente en la alimentación y en la medicina tradicional. En 1980 fue hecho el análisis de contenido graso y proteico realizado en el Instituto de Ciencia de los Alimentos de la Universidad Cornell en USA y por Duclos en Florida, demostrando que las semillas de Plukenetia volubilis L., contienen $48 \%$ de aceite y $29 \%$ de proteínas. Asimismo, en el año 1992 se reportó una concentración del $54 \%$ de aceite y $27 \%$ de proteína a través de un análisis de aminoácidos esenciales y no esenciales que fue comparado con oleaginosas recomendadas por la FAO, WHO \& ONU para la alimentación, mostrando elevadas concentraciones de cisteína, tirosina, treonina y del triptófano equivalente a otras proteínas de semillas oleaginosas de la región y un contenido ligeramente bajo en lisina y leucina; además de un alto contenido de aceite linoleico y linolénico (Aranda Ventura, 2009).

Ya el en año 2003, se caracterizó el aceite de Sacha Inchi, aplicando técnicas de espectrofotometría de infrarojo por transformado de Fourier -FTIR y Resonancia Magnética Nuclear de protón; H1 NMR (por sus siglas en inglés), en comparación con el aceite de linaza; llegando a la conclusión de que el aceite de Sacha Inchi es fuente de w-3(ácido linolénico) y w-6(ácido linoleico) y, de acuerdo a Sotero, en una investigación con semillas seleccionadas, obtuvo una concentración de $41,23 \mathrm{mg} / 100 \mathrm{~g}$ de tocoferoles, presentando buena estabilidad cuando es almacenado por seis meses a temperatura ambiente controlada (Bandoli P, 2006).

Se concluye que el aceite de Sacha Inchi es muy rico en ácidos grasos insaturados (93\%), de gran importancia para la nutrición por su alto contenido de ácidos grasos esenciales, en un 84\%. Con 36\% de linoléico Omega-6 y 48\% de alfa-linolénico (Omega-3), este último contribuye al equilibrio del colesterol, principal causa de mortalidad en el mundo; facilita la microcirculación de la sangre y la irrigación cerebral, previene los accidentes cardiovasculares e incluso los infartos y los Omegas contenidos, cumple una función fisiológica de elongación, es decir, la producción de ácido eicosapentanoico (EPA) y ácido 
docosahexaenoico (DHA) en cantidad suficiente para todas sus necesidades biológicas (Crawford MA, 1992). De este modo, el Sacha Inchi tendría efecto hipolipemiante. Es por esta razón que se plantea evaluar los beneficios hipolipemiantes del Sacha Inchi en pacientes con hipercolesterolemia e hipertrigliciridemia en un grupo de adultos que viven en el Asentamiento Humano Nueva Alianza.

\section{MATERIAL Y MÉTODOS}

Diseño Preexperimental: este tipo de diseño administra un tratamiento o estímulo en la modalidad de una preprueba y posprueba, además utiliza un grupo, por ende no existe la posibilidad de comparación de grupos. De corte longitudinal prospectivo: analiza cambios a través del tiempo en determinadas variables y recolecta datos a través del tiempo en puntos o períodos específicos para hacer inferencias en los cambios determinates y consecuencias.

\section{Tabla 1}

Índice de Masa Corporal de los participantes

\begin{tabular}{ccc}
\hline & N & $\%$ \\
\hline $18.5-<25$ Normal & 2 & 6,7 \\
$25-30$ Sobrepeso & 8 & 26,7 \\
$30-35$ Obesidad & 20 & 66,7 \\
Total & 30 & 100,0 \\
\hline
\end{tabular}

Fichas de recolección de datos

- Ficha clínica.

- Ficha de control de cumplimiento de la ingesta del aceite de Sacha Inchi.

- Ficha recordatoria de alimentos.

Obtención del aceite de Sacha Inchi

Fue adquirido de Laboratorios Olivos del Sur

\section{Dosis para el consumo diario}

La dosis de consumo del aceite de Sacha Inchi (requerimiento diario admitido) de Omega-3 para una persona entre las edades 35 y 64 años es del $1.5 \%$ del valor calórico total del día, teniendo como base una dieta de 1700 calorías, según el recordatorio de alimentos realizados a los participantes. fue $45 \mathrm{ml}$ durante 42 días; sabiendo que el RDA
Alcance explicativo: indica las causas de los eventos, sucesos o fenómenos estudiados, explicando las condiciones en las que se manifiesta (Hernández, 2000).

\section{Población y muestra}

Son 30 participantes, entre las edades de $35 \mathrm{y}$ 64 años, de los cuales son 20 de sexo femenino y 10 de sexo masculino. Que fueron captados del registro del área de nutrición con diagnóstico de hipercolesterolemia e hipertriglicéridemia del Centro de Salud Miguel Grau, a quienes se les visitó en su vivienda y se les explicó e informó de los beneficios y los riesgos inherentes a su participación, de acuerdo a las normas éticas de investigación de la Universidad Peruana Unión y se procedió, finalmente, a firmar el consentimiento informado, si es que cumplían con los criterios de inclusión de la investigación.

\section{Procedimiento}

Firmar el consentimiento informado, si es que el participante cumple con los criterios de inclusión. Se informó, a cada participante, los efectos benéficos del aceite de Sacha Inchi y probables riesgos de su participación en el estudio, asimismo, completar los datos de la ficha clínica. Se les realizó la prueba bioquímica del perfil lipídico para iniciar el consumo del aceite de Sacha Inchi. Se entregó una botella de aceite de Sacha Inchi para cada semana cumpliendo las 6 semanas del tratamiento.

Se monitorizó el consumo del aceite de Sacha Inchi, de la siguiente manera:

Cada semana recibieron 4 visitas domiciliarias a cada participante, con el fin de asegurar el cumplimiento de la ingesta y, además, se le hizo un seguimiento telefónico durante el día. Las investigadoras monitorizaron la ingesta del aceite de 
Sacha Inchi mediante el registro de cumplimiento de consumo diario, el cual se aplicaba en cada visita.

Cada domingo, de las semanas, se entregaba una nueva botella de aceite de Sacha Inchi.

Finalmente, se realizó la última prueba del perfil lipídico, y se procedió a informar los resultados a cada participante.

Con el propósito de contrastar las hipótesis planteadas, se ha procedido a realizar, primero, la prueba de normalidad para precisar si las variables presentan una distribución normal antes y después del tratamiento. En ese sentido se presenta los resultados de la prueba de normalidad de Kolmogorov-Smirnov (K-S) y se muestra los datos correspondientes a las 4 variables dependientes, resaltando que todos presentan una distribución normal, dado que el $\mathrm{p}$ valor obtenido para todos los casos es mayor que 0.05 . Por lo tanto, para los análisis estadísticos de comparación entre el pre test y pos test se realizará mediante la $\mathrm{T}$ de Student. En la tabla se muestra la prueba de normalidad de los componentes del perfil lipídico.

Tabla 2

Prueba de Normalidad de los componentes del perfil lipídico

\begin{tabular}{cccccc}
\hline \multirow{4}{*}{ Antes } & Medía & D.E & K.S & P \\
\hline \multirow{7}{*}{ Después } & Colesterol total & 264.41 & 34.469 & .657 & .781 \\
& Triglicéridos & 219.99 & 42.02 & .723 & .673 \\
& Col_HDL & 39.69 & 5.729 & .450 & .988 \\
& Col_LDL & 198.4 & 43.85 & 1.063 & .209 \\
& Colesterol total & 174.21 & 15.754 & .806 & .535 \\
& Triglicéridos & 137.37 & 8.1555 & .851 & .465 \\
& Col_HDL & 63.283 & 3.0620 & .861 & .449 \\
& Col_LDL & 135.24 & 7.8019 & .573 & .898 \\
\hline
\end{tabular}

\section{RESULTADOS}

Tabla 3

Diferencias significativas del nivel de colesterol total, antes y después de la intervención, en las personas entre las edades de 35 y 64 años con diagnóstico de hipercolesterolemia e hipertrigliceridemia.

\begin{tabular}{cccccc}
\hline Nivel de colesterol total & Media & Desviación Estándar & $\mathrm{T}$ & $\mathrm{gl}$ & $\mathrm{p}$ \\
\hline Antes de la intervención & 264,41 & 34,469 & \multirow{2}{*}{17,02} & \multirow{2}{*}{29} & \multirow{2}{*}{, 000} \\
Después de la intervención & 174,21 & 15,75 & & & \\
\hline
\end{tabular}

En la tabla 3, se muestra que existen diferencias significativas antes y después de la ingesta de Sacha Inchi en el nivel de colesterol total de los participantes $(\mathrm{t}=17,02 ; \mathrm{p}<.05)$, asimismo, se aprecia que el nivel de colesterol total, después del consumo, ha disminuido significativamente $(\mathrm{x}=174.21)$. Entonces el consumo de aceite de Sacha Inchi durante 42 días logra reducir los niveles de colesterol total.

Tabla 4

Diferencias significativas del nivel de colesterol LDL, antes y después de la intervención, en las personas entre las edades de 35 y 64 años con diagnóstico de hipercolesterolemia e hipertrigliceridemia.

\begin{tabular}{lccccc}
\hline & Media & D.E & T & gl & P \\
\hline Nivel de colesterol LDL & & & & & \\
Antes de la intervención & 198,44 & 43,85 & 8,46 & 29 &, 000 \\
Después de la intervención & 135,24 & 7,80 & & & \\
\hline
\end{tabular}


En la tabla 4, se muestra la diferencia significativa antes y después del consumo del aceite de Sacha Inchi en el nivel del Col - LDL $(t=8,46 ; p<.05)$, se observa que el nivel de Col - LDL ha disminuido significativamente $(\mathrm{x}=135,24)$. Entonces la ingesta de aceite de Sacha Inchi, durante 42 días, logra reducir los niveles de Col - LDL.

Tabla 5

Diferencias significativas del nivel de colesterol HDL, antes y después de la intervención, en las personas entre las edades de 35 y 64 años con diagnóstico de hipercolesterolemia e hipertrigliceridemia.

\begin{tabular}{lccccc}
\hline Nivel de Colesterol HDL & Media & D.E & T & gl & P \\
\hline Antes de la intervención & 39,69 & 5,72 & & & \\
Después de la Intervención & 63,28 & 3,06 & 19,13 & 29 & \multirow{2}{*}{000}
\end{tabular}

Se observa, en la Tabla 5, la diferencia significativa antes y después del consumo del aceite de Sacha Inchi en el nivel del Col - HDL $(t=19,13 ; p<.05)$, se muestra que el nivel de Col - HDL, después del consumo, ha aumentado significativamente durante las 42 días del tratamiento $(\mathrm{x}=63,28)$.

Tabla 6

Diferencias significativas del nivel de triglicéridos, antes y después de la intervención, en las personas entre las edades de 35 y 64 años con diagnóstico de hipercolesterolemia e hipertrigliceridemia.

\begin{tabular}{cccccc}
\hline Nivel de Triglicéridos & Media & D.E & T & gl & P \\
\hline Antes de la intervención & 219,99 & 42,02 & \multirow{2}{*}{10,54} & \multirow{2}{*}{29} & \multirow{2}{*}{000} \\
Después de la intervención & 137,37 & 8,15 & & & \\
\hline
\end{tabular}

Se observa, en la tabla 6, que existe diferencia significativa antes y después de la ingesta del aceite de Sacha Inchi en el nivel de triglicéridos de los participantes $(\mathrm{t}=10,54 ; \mathrm{p}<.05)$, asimismo, se aprecia que el nivel de triglicéridos, después del consumo, ha disminuido significativamente $(\mathrm{x}=137,37)$. Entonces el consumo del aceite de Sacha Inchi, durante 42 días, logra reducir los niveles de triglicéridos.

\section{DISCUSIÓN}

Al término de los 42 días, administrando $45 \mathrm{ml}$ de aceite de Sacha Inchi a las personas consideradas en la muestra con diagnóstico de hipercolesterolemia e hipertrigliceridemia, se ha podido verificar comparando con los datos bioquímicos tomados antes y después del tratamiento que existen diferencia significativa después del consumo del aceite de Sacha Sinchi, observándose que disminuyeron los niveles de triglicéridos, colesterol total, colesterol LDL y aumento de colesterol HDL.

En los parámetros evaluados, los niveles de triglicéridos disminuyeron en un promedio (antes 219.99- después 137.37), por ende, aumentó los niveles de colesterol- HDL en un promedio (antes
39,69- después 63,28). Este coincide con un estudio realizado por Vicuña y cols, quienes demostraron el efecto del aceite de "Sacha Inchi" en rattus rattus albinus con hipertrigliceridemia experimental, lográndose una reducción, a las dos semanas, del $45,57 \%$, comparado con el gemfibrozilo de 44,83\% y el control de $27,24 \%$, concluyendo que el efecto se mostró en un $20 \%$ a la tercera semana, sin embargo, se mantuvo constante en el transcurso de la tercera a la sexta semana, por lo que se necesitaría un mayor tiempo de tratamiento, para asegurar su efecto (Vicuña A, 2009).

Asimismo, el estudio de (Huaman J, 2008) encontró resultados favorables en la disminución de los triglicéridos en donde se administró $50 \mathrm{~g}$ de semillas de Sacha Inchi a adultos jóvenes aparentemente sanos, sometidos a una prueba de tolerancia a triglicéridos. En esta investigación, al graficar la prueba de tolerancia a triglicéridos, se evidenció significancia estadística al comparar el área bajo la curva de ambas fases, con una disminución de $38.17 \%$ con el consumo de Sacha Inchi.

Otro estudio realizado por el Cómite de National Cholesterol Education Program (NCEP) comparó el efecto de las estatinas, logrando una disminución sobre los triglicéridos entre 7 y $30 \%$, mientras que los fibratos disminuyen los triglicéridos entre 20 
y $50 \%$, por ende dedujo el Camec Iquitos, en un estudio que realizaron sobre el efecto hipolipemiante en los TG, teniendo como resultado una disminución neta de $(21.82 \%)$, estando este dentro del rango hipolipemiante de estos fármacos, por este motivo se consideró en este estudio que el aceite de Sacha Inchi ejerce un efecto hipolipemiante para el control de las hipertrigliceridemias.

Con respecto a los niveles de colesterol HDL, aumentaron automáticamente después de las 6 semanas de tratamiento, y esto se debe al contenido rico en omega 3; dentro de todas las fuentes naturales conocidas, es el que mayor contenido posee. En comparación a los aceites de todas las semillas oleaginosas utilizadas en el mundo, para consumo humano, es el más rico en ácidos grasos insaturados; posee entre $45,2 \%$ y $48,6 \%$ de ácido graso esencial alfa linolénico omega 3 y tiene el contenido más bajo de ácidos grasos saturados, $6,39 \%$ en promedio, $3,85 \%$ de palmítico y $2,54 \%$ de esteárico.

Asimismo, es importante considerar las ventajas de su gran disponibilidad, menor costo y mayor concentración de omega-3, comparado con 200gr. de "jurel" que contienen 3,4gr. de omega-3 y esa misma cantidad se obtendría con $15 \mathrm{gr}$. de aceite de "Sacha Inchi"108, este disminuye los lípidos plasmáticos ejerciendo su efecto hipolipemiante, al parecer por los Omegas, actuando con sus diferentes mecanismos: aumento de la depuración de los quilomicrones y triglicéridos por incremento de la actividad lipasa lipoproteica disminución de la síntesis de TG y de la secreción de VLDL y aumento de la beta oxidación de los ácidos ${ }^{109}$, grasos en el hígado. Además, los ácidos grasos omega-3 son activadores del PPAR alfa, especialmente el ácido docosahexaenoico (DHA, 22:6 n-3) que se deriva del ácido alfa-linolénico, presentándose, en un 48,61 \%, en el aceite de "Sacha Inchi”.

Podemos concluir que el efecto hipolipimiante, del aceite de Sacha Inchi, probablemente se deba al omega 3, por ser uno de sus componentes químicos mayoritarios aumentando los niveles bajos de colesterol HDL, de acuerdo con los resultados obtenidos en nuestra investigación, esto coincide con un estudio realizado por Ramón y cols ${ }^{111}$, quienes usaron, como fuente de omega-3, el pescado azul en personas normales como en pacientes con niveles de colesterol HDL bajos, concluyendo que la ingesta mostró a las 6 semanas un aumento de $30.2 \%$ y en el 3er mes $47.71 \%$, asimismo, Harris, Connor y cols ${ }^{112}$, mostraron que después de 4 semanas, con una dieta rica de salmón, encontraron un aumento del $50 \%$ en el colesterol HDL.
Asimismo, los niveles de colesterol-total disminuyeron en un promedio (antes 264.41después 174.21) y colesterol LDL (antes198.44despues135.24), esto coincide con un estudio realizado en Camec Iquitos, en un grupo control y grupo experimental disminuyó el colesterol total en $26.61 \%$, mientras que el grupo control solo disminuyó en $6.42 \%$, podemos decir que la disminución de colesterol total, atribuida al aceite de Sacha Inchi sería $20.19 \%$, el resto sería explicado por el efecto de los cambios terapéuticos de estilo de vida. Este valor está dentro del rango de disminución neta de colesterol total (16.8 -26.56\%) logrado, al administrar aceite de Sacha Inchi en ratas Holtzman hiperlipidémicas con un peso promedio de $251 \mathrm{~g}$; este estudio evaluó dosis de 200, 400, 800, 1600 y $3200 \mu \mathrm{l}$ de aceite de Sacha Inchi por día durante 5 semanas ${ }^{113}$. este efecto rápido es semejante al del aceite de Argan (Argania spinosa L.) el cual logró una disminución del 36.67\% del colesterol total en solo 7 semanas y asimismo en colesterol LDL.

El grupo de participantes que consumió $45 \mathrm{ml}$ de aceite de Sacha Inchi o Plukenetia volubilis Linneo, durante 6 semanas, logró disminuir los valores de: colesterol-total, colesterol-LDL, triglicéridos y aumentando los niveles de colesterol-HDL.

\section{Declaración de financiamiento y de conflicto de intereses:}

El estudio fue financiado por los autores, quienes declaran no tener algún tipo de conflicto de interés en la investigación realizada.

\section{Correspondencia:}

\section{María Gamarra Camacho}

Jefa de Laboratorio de Nutrición. Universidad Peruana Unión. Carretera Central Km 19.5 Ñaña. Lima. Perú.

e-mail: lulugc_5@hotmail.com 


\section{REFERENCIAS BIBLIOGRÁFICAS}

Aranda Ventura, V. J. (2009). Composición de ácidos grasos del aceite extraído de seis muestras de semillas de Plukenetia VolúbilisL. Etnomedicina - IMET- EsSalud, $1-2$.

Bandoli P, D. L. (2006). Alpha linolénic acid rich oils, Composition of Plukenetia Volúbilis Oil from Perú. Italiana Delle Sostanze, 120-123.

Bussman R, T. C. (2009). Plukenetia huayllabana. Nord J. Bot volumen 27, 313-315.

Carmena, R. (s.f.). Recomendaciones generales para el tratamiento de la dislipidemia. Departamento de Medicina.

Cordova M, C. J. (2008). Evaluación de la toxicidad aguda y acción hipolipemiante del aceite de sacha inchi, Facultad de Medicina Humana de la Universidad de San Martín de Porres. Horizonte Médico, 50-54.

Correo, J., \& Yesid, H. (2007). Corporación Andina de Fomento CAF. SECAB; Ministerio de Educación y Ciencias de España, 577-596.

Crawford MA, C. K. (1992). Phylactos A. Essential fatty acids in early development. In: Polyunsatured fatty acids in human nutrition. New York : Raven Press, 93 109. gamarra, 1., \& floresz, p. (2006). lipoli9mia. educ, $12-30$.

Gorriti A, A. J. (2010). Toxicidad oral a 60 días del aceite de sacha inchi (Plukenetia volúbilis Linneo) y Linaza
(Linum ussutatissimum) y determinación de la dosis letal 50ml en roedores. Rev Perú Med Exp Salud Pública, 27- 60.

Harris WS, C. W. (1983). The comparative reductions of the plasma lipids and lipoproteins by dietary polyunsaturated fast: salmon oil versus vegetables oils Metabolism. Española Cardiovascular, 32-84.

Hernandez, F. y. (14 de junio de 2000). es. slideshare.net/ solanghy/diseo preexperimental 42988863.

Huaman J, C. K. (2008). Efecto de la Plukenetia volúbilis Linneo (sacha inchi) en la trigliceridemia posprandial. Fac med, 69-78.

NCEP. (2001). Expert panel of detection, evaluation and treatment of high blood cholesterol in adults. Executive summary of the third report of the National Cholesterol Education Program, 2486-2497.

Ramón Segura, C. J. (2007). Los ácidos grasos Omega-3 en la promoción de la salud y la prevención de las enfermedades crónicas. Española de Obesos, 5 - 39.

Simopoulos. (2008). The importance of the omega-6/ omega acid ratio in cardiovascular disease and other chronic diseases. Exp Biol Med, 674-688.

Vicuña A, I. E. (2009). Gemfibrozilo vs aceite de sacha inchi en la reducción de niveles de triglicéridos séricos en rattus albinus. XXII Congreso Científico Nacional. España volumen II, 56-57.

Recibido: 18/01/2015 Aceptado: 19/05/2015 